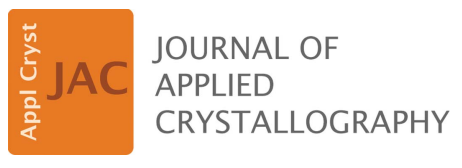

ISSN 1600-5767

\title{
A new library of 3D models and problems for teaching crystallographic symmetry generated through Blender for use with 3D printers or Sketchfab
}

\author{
Michael M. Aristov, ${ }^{*}$ Han Geng, Alex Pavelic and John F. Berry*
}

Department of Chemistry, University of Wisconsin-Madison, 1101 University Avenue, Madison, WI 53706, USA. *Correspondence e-mail: aristov@wisc.edu, berry@chem.wisc.edu

Edited by S. Moggach, The University of Western Australia, Australia

This work is dedicated in memory of Professor Marilyn M. Olmstead and Professor Larry F. Dahl, two of the most passionate crystallographers and educators these authors ever had the pleasure of learning from and working with. May their influence live on forever in the countless crystallographers that they have fostered.

Keywords: 3D models; 3D animations; education.

Supporting information: this article has supporting information at journals.iucr.org/j

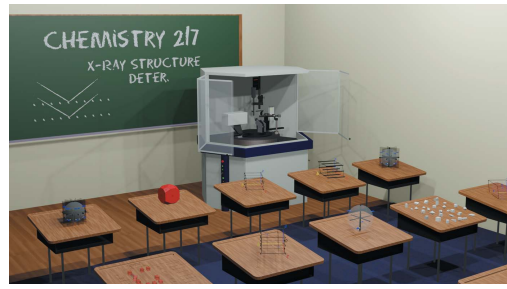

A new and growing library of 3D models that can be utilized to illustrate many important concepts in the field of crystallography is presented. These models are accessible in the classroom via computers and smartphones and offer significant advantages over 2D depictions found in crystallography textbooks. Through the use of Blender, a free 3D modeling and animation program, over 100 new models focusing on different aspects of crystallographic education have been created. To simplify distribution/access, all of these models have been uploaded to Sketchfab, a model hosting and viewing web site that works similarly to YouTube. The current set of models is also given as a list in the supporting information. All of these models are free to view in a web browser or through a smartphone application. Additionally, all of these models are freely downloadable through the supporting information and Sketchfab, and users are encouraged to download and modify these models to best suit their needs. This library of models is part of the authors' ongoing outreach program to provide 3D models for free for educational purposes, and the authors offer their services to create additional models and moderate this library as additional requests or critiques are provided.

\section{Introduction}

By its very nature, understanding crystallography requires a strong ability to intuit 3D spatial relationships (Chapuis, 2011; Gražulis et al., 2015). These relationships can be taught to students via mathematical concepts such as matrix algebra, symmetry and group theory, though diagrammatic demonstration of symmetry relationships is of prime importance and utility (Tuvi-Arad \& Blonder, 2010; Grafton, 2011; Luxford et al., 2012; Southam \& Lewis, 2013; Fuchigami et al., 2016; Girolami, 2016; Penny et al., 2017; Ruiz \& Johnstone, 2020). Classically, 2D projections (stereographic projections) of 3D relationships have been used in crystallography (Hardgrove, 1997; Stróż, 1997; Chen et al., 2015; Duda et al., 2020). Over time, teaching of molecular symmetry has improved significantly because of the work of educators to create either physical or virtual libraries of 3D models, including some with 3D printing capabilities (Scalfani \& Vaid, 2014; Casas \& Estop, 2015; Rodenbough et al., 2015; Penny et al., 2017; Casas, 2018, 2020; Savchenkov, 2020), to aid in the visualization of this inherently 3D topic (Graef, 1998; Stróż, 2003; Hitzer et al., 2010; Tuvi-Arad \& Blonder, 2010; Antonoglou et al., 2011; Flint, 2011; Carlisle et al., 2015; Johnston, 2021). These libraries capitalize on visualization software such as $\mathrm{Jmol}$, allowing users to manipulate 3D structures through the use of a mouse and the integration of 3D models into web pages 
(Hanson et al., 2013). Examples of libraries of 3D models on chemistry topics that are now used across campuses include Otterbein Symmetry, the Orbitron and other education libraries on Sketchfab (Aristov et al., 2021; Johnston, 2021; Winter, 2021). While these libraries are excellent resources for visualizing inherently $3 \mathrm{D}$ concepts like molecular orbitals or molecular symmetry, more specialized topics like crystallographic symmetry, including translational symmetry and space symmetry, fall outside of their original scope (Tuvi-Arad \& Blonder, 2010; Antonoglou et al., 2011; Flint, 2011; Southam \& Lewis, 2013; Carlisle et al., 2015; Penny et al., 2017; Dagnoni Huelsmann et al., 2018). Although there are still numerous resources that focus on teaching crystallography (Grove et al., 2009; Cushman \& Linford, 2015; Malbrecht et al., 2016; Bazley et al., 2018), few fully incorporate three dimensionality (Scalfani \& Vaid, 2014; Casas \& Estop, 2015; Rodenbough et al., 2015; Brannon et al., 2020).

We report here a large new library of 3D models and animations expressly designed for the purpose of teaching crystallography. Herein, we define a model as any threedimensional representation or animation that can be manipulated and viewed in three dimensions. In creating these models, we have paid careful attention to converting the historically used 2D projections found throughout textbooks (Meier, 1984; Borchardt-Ott, 1993; Graef, 1998; Meier, 2001; Girolami, 2016) and International Tables for Crystallography (2016, hereafter International Tables), Vol. A, into recognizable 3D counterparts. We have sought to make this library easily and globally accessible, barrierless, free, and expandable/curatable. To accomplish these goals, we make use of Sketchfab, a free web site for hosting, editing and viewing 3D models (https://sketchfab.com/). All of the models are freely available on the Sketchfab web site, which can be viewed either in an internet browser on all operating systems or through a smart phone application available on either iOS or Android. As the models can be viewed inside a web browser through Sketchfab, users do not need to download or learn additional software packages to make use of the models. All primary functions for viewing these models on Sketchfab are free, thereby avoiding the potential financial burden that other teaching tools, like physical model kits or proprietary software packages, put on students. Additionally, anyone can freely add their own models to Sketchfab, and if they include specific tags, like Crystallography_education or Crystallography_activities or Stereographic_ Projection, these models can be easily found alongside the initial library we describe here. New models can be curated through feedback via the comment section or by contacting the author, allowing the community to help self-moderate.

The models themselves were made using Blender (https:// www.blender.org), a free 3D modeling software program that is compatible with most research-grade software packages, including Chimera, Mercury and PyMol (Pettersen et al., 2004; Macrae et al., 2020; Schrödinger, 2021). The Blender guides by Dr Joseph G. Manion (2021) proved to be exceptionally useful for making many of these models and using Blender in general. To help determine the scope of models to make, we

communicated with groups of crystallography teachers and students. Currently, this library contains over 130 models/ animations, including models showing the action of symmetry operations, depictions of point and space symmetry, and illustrations of unit cells from various crystal systems. Although we have constructed a robust set of models for these topics, we plan to expand upon this library as we receive additional nuanced feedback or model requests on previously unconsidered topics.

\section{Technical considerations}

\subsection{General model settings}

All models uploaded to Sketchfab were made with the following specifications. All models had their field-of-view (FOV) set to $1^{\circ}$, which is an option built into Sketchfab's 3D model editor. This FOV was selected to avoid issues with parallax and minimize distortion during reorientations. The models' shadows were turned off to minimize unnecessary visual information during reorientation of the camera. Either the default, off-white (\#dddddd) or the included 'clean_dark'/ 'clean_light' image backgrounds were used depending on which one provided clearer visualization of the model.

\subsection{D symbols for symmetry elements}

Adapting the well known 2D symbols from stereographic projections to $3 \mathrm{D}$ visualization aids proved to be non-trivial. We primarily referenced X-ray Crystallography (Girolami, 2016) and International Tables to construct 3D versions of the historical 2D representations for the symbols. While some symbols, such as the rotational axes or mirror planes, had intuitive 3D counterparts, others, like the inversion center or glide planes, did not. For mirror planes we used a flat, gray, transparent 2D surface. For rotation axes, we opted for a matte black bar whose end-on profile matches that of the $2 \mathrm{D}$ projection. For inversion centers we used a small metallic red

Symmetry Symbols for 2D Projections

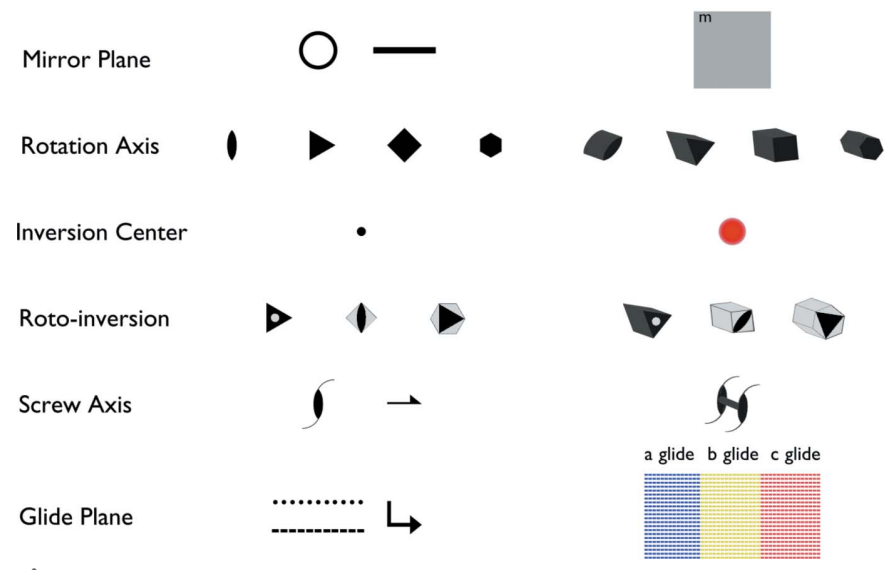

Figure 1

A comparison between the 2D symmetry symbols used in stereographic projections and our $3 \mathrm{D}$ interpretation of those symbols. 
sphere. If another symmetry element passed through an inversion center, we cut a hole through the secondary symmetry element, leaving a void space around the inversion center so that it was not obscured. For roto-inversion axes, we added a white dot to the end-on profile of $\overline{3}$, whereas $\overline{4}$ and $\overline{6}$ were drawn as white tetragonal/hexagonal prisms with a black twofold and threefold rotation symbol imposed on their faces, respectively. For unit cells, we color coded the axes such that a was blue, b was yellow and c was red. Glide planes were colored according to the direction of translation, for instance a $c$ glide, regardless of whether it is perpendicular to $\mathbf{a}$ or $\mathbf{b}$, was colored red. The $n$-glide planes were colored according to the combination of translations: e.g. an $n$ glide along a and $\mathbf{c}$ was colored purple (blue + red). Examples of these elements can be seen in Fig. 1.

\subsection{D symbols for objects}

This library contains two primary types of objects to be acted upon by symmetry elements. These objects include the Blender default 'Suzanne' model, protected under the Creative Commons Attribution 3.0 un-ported license. The second object is a custom-made blue hand holding a yellow coin designed to help emphasize chirality (Fig. 2). These 'hands' are inspired by W. M. Meier's (1984) pamphlet for teaching crystallographic space groups.

\subsection{Static versus animated}

One benefit from creating these models in Blender is the ease with which animations could be made from the models. This process, although technically simple through Blender's included options, allows for the generation of animations depicting all of the symmetry operations used in crystallography. Models including animations can be identified easily on the Sketchfab web site by their thumbnail showing a small depiction of film in the upper right corner on the animated models.

\subsection{Availability and expandability}

All of the models that we have made are freely available on Michael Aristov's Sketchfab account at https://sketchfab.com/ Michael.Aristov. All models relevant to crystallography are tagged with either Crystallography_education or Crystallography_activities to make them more visible to the Sketchfab search engine. These models can be viewed either in-browser or through the free Sketchfab

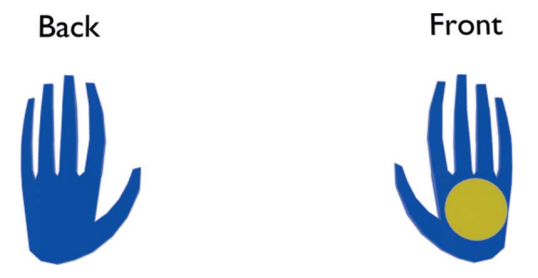

Figure 2

A depiction of the model we chose for the chiral object, showing both the back and the front of the left hand. smartphone application available on both Android and iOS (https://sketchfab.com/). To search for a tag on Sketchfab, the user needs to type tag: at the start of their search. Additionally, users need to take care when typing in a tag that they do not use a space (' ') instead of an underscore ('_'), as tags do not contain spaces. All models described in this publication can also be downloaded from the supporting information (SI). The SI contains duplicate models in a variety of file formats depending on their purpose. To make these models easier to navigate, we have provided direct links throughout this paper. Additionally, the SI includes an Excel spreadsheet that contains an interactive link to every model and for every tag search relevant to this project. We encourage students and teachers to download, modify and reupload these models while including the Crystallography_education or Crystallography_activities tag as appropriate to help expand this library and provide additional resources for crystallography topics we may have missed.

\subsection{File types}

3D file formats have a variety of file types, each with their own specifications and capabilities. All of the models were originally made in Blender and are therefore available as . blend files. Models created for 3D printing are available as .st 1 files. Additionally, the files uploaded to Sketchfab can be downloaded directly as their original upload file type, as a .blend, or as . glTf, a universal 3D file format, or USDZ, an augmented reality file type. For animation purposes, the $3 \mathrm{D}$ space-group problems were uploaded as paired .obj/.mtl files, which contain the $3 \mathrm{D}$ coordinates and materials, respectively.

\subsection{D printing}

3D printing is rapidly becoming accessible on most university campuses. The capability for 3D printing trivializes the distribution of physical teaching tools, as it eliminates the need for costly shipping. All models in this paper intended for $3 \mathrm{D}$ printing are available in the SI as . st I files. To reduce the cost of $3 \mathrm{D}$ printing, each model was made hollow to minimize the amount of $3 \mathrm{D}$ printing material required. These models can and will need to be scaled to fit the large variety of commercially available $3 \mathrm{D}$ printers.

\section{Discussion}

\subsection{A need for new objects}

For point-group symmetry in International Tables, Vol. A, Table 3.2.3.1, open circles and dots are used to denote object(s) above or below the projection plane, respectively. Furthermore, the space-group diagrams implement circles, or circles with an inscribed comma (to denote a change in handedness), to represent symmetry operations on the asymmetric unit. While functional at showing the symmetry, the positions of these objects and a change in chirality, this notation does not indicate directionality. Translating the circles from 2D projections into spheres for 3D models creates 
certain ambiguities as a sphere is too symmetric to serve as a proper placeholder for an 'asymmetric unit'. An example of ambiguous symmetry operations arising from a set of these symmetric symbols is shown in Fig. 3. In the 2D projection (Fig. 3, top), a coordinate system with a symmetry axis normal to the plane is set by the nature of the projection, and students can easily recognize the $(x, y, z) \rightarrow(\bar{x}, \bar{y}, \bar{z})$ relationship between the two points, one open, one filled. When translating this exercise to three dimensions, however, the two points are represented with identical black spheres, and students are able (and encouraged) to rotate the model in any way that they like. When small black spheres are used to denote the asymmetric unit, students are rightly able to see that the two highly symmetric objects could be related to each other by either a twofold axis, a mirror plane or an inversion center. The 2D projection that International Tables uses in Table 3.2.3.1 to denote the point group $\overline{1}$ actually has $\frac{\infty}{m} m$ symmetry when translated literally into three dimensions as a direct result of spherically symmetric objects being used. Unambiguous 3D exercises for symmetry determination require asymmetric chiral objects instead of achiral spheres, whereby the symmetry operations for any point symmetry can be unambiguously determined from the arrangement of the objects alone. For this reason, we developed these 3D renderings using the chiral hand objects introduced in Fig. 2.

\section{The 3D library}

\subsection{General considerations}

The models in the library described throughout this paper fall into two general classes - 3D visualization aids and 3D

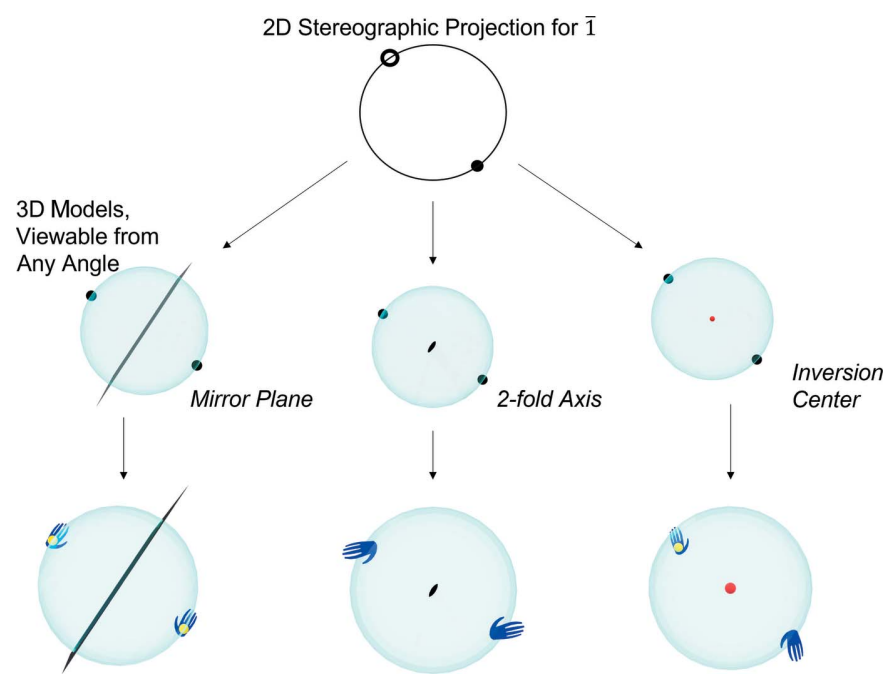

Figure 3

A depiction of how a set of the symmetric circle symbols generated from the objects used in International Tables, Vol. A, Table 3.2.3.1, for pointgroup symmetry can give rise to multiple, equally correct, interpretations of the absent symmetry operation when translated to three dimensions as compared to the 3D models with full asymmetric objects. By using the asymmetric hand object, ambiguity is removed and the three different symmetry operations implied by the spheres now give rise to unique diagrams (bottom). activities. The visualization aids provide students with a 3D representation for many of the inherently 3D concepts necessary to understand crystallographic symmetry. The 3D activities allow students to apply the understanding that they garner from the visualization aids through problem-solving activities. Topics pertain to specific frequently raised student questions or common student misconceptions. Some of these topics include why the stereographic projections for cubic groups have curved lines for mirror planes, the difference between $4_{1}$ and $4_{3}$ screw axes, and the obverse versus reverse rhombohedral centerings. This library of models capitalizes on our existing experience in combining Blender, chemistry education and Sketchfab (Aristov et al., 2021). These models are further described in Table 1.

\subsection{Example models}

Symmetry is a foundational topic in crystallography, and its presentation differs from how the topic is introduced in chemistry courses. The use of Hermann-Mauguin definitions in crystallography as opposed to Schönflies definitions in the chemistry context can cause potential confusion for students. A striking example is the handling of improper rotation axes, which are defined as roto-inversion axes in the HermannMauguin system and as roto-reflections in the Schönflies system. To help teach this topic, we created a model for each of the 32 crystallographic point groups. These models contain a semi-transparent sphere to represent the stereographic projection of the objects, the hands with coins to represent the chiral objects, and the various symmetry elements representative of the group. For space groups, we have modeled many of the lower-symmetry systems. These include all triclinic and monoclinic as well as some selected highersymmetry systems. Each of these models by default has colorcoded axes and a black outline to denote the unit cell, and the hands with coins are used to represent the chiral object. Additionally, by switching from 'static pose' to 'display symmetry elements', an option available in the bottom left of the user interface on the browser-based Sketchfab web site, the various symmetry elements representative of the group will be displayed. Example images of these types of 3D depictions can be found in Table 1.

\subsection{Crystallographic activities}

Many of the activities provided in this 3D library were created with the intent for students to tackle them collaboratively in small groups of 3-4, and many have been tested successfully in this mode. The versatility of Sketchfab allows students to easily access the $3 \mathrm{D}$ models either on smartphones through the official Sketchfab application or through a provided web browser link. These links can be emailed to the students beforehand, added to a course web page or built into printable QR codes. The specific problems and student expectations are described below.

4.3.1. Unit-cell problems. The unit-cell problems each contain a single labeled unit cell that is initially randomly oriented and can be reoriented subsequently through student 
Table 1

Selected models currently freely available on Sketchfab.

Each entry is accompanied by the model's title as found on Sketchfab, a direct link, a short description, and a snapshot of the model or animation.

\begin{tabular}{ll}
\hline & Description \\
\hline $\begin{array}{l}\text { Symmetry elements } \\
\text { Crystallographic translational }\end{array}$ & $\begin{array}{c}\text { An animation showing how translation } \\
\text { symmetry (orth): }\end{array}$ \\
generates symmetry-related objects in \\
& $\begin{array}{l}\text { adjacent unit cells in an orthorhombic } \\
\text { system. }\end{array}$
\end{tabular}

Crystallographic translational symmetry (tri):

https://skfb.ly/o7YQo

An animation showing how translation

Crystallographic rotation axes: An animation comparing the two-, three-, https://skfb.ly/o7YPZ

Symmetry: mirror plane: https://skfb.ly/o7YPX generates symmetry-related objects in adjacent unit cells in a triclinic system. four- and sixfold rotational symmetry axes side by side. This animation shows how the operation generates the symmetry-related objects.

An animation showing how a mirror plane operates on an object to generate its symmetry-related counterpart.

Snapshot
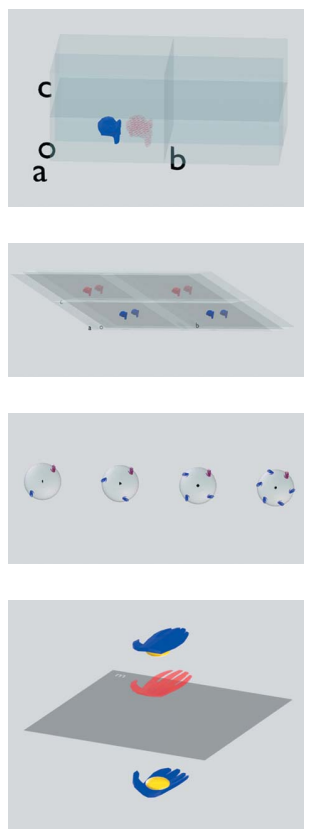

An animation showing how an inversion center operates on an object to generate its symmetry-related counterpart.
Symmetry: inversion center: https://skfb.ly/o7YQu

Symmetry: roto-inversion: https://skfb.ly/o7YQx https://skfb.ly/o7YQC

$4_{1}$ and $4_{3}$ screw axes: https://skfb.ly/o7YQE

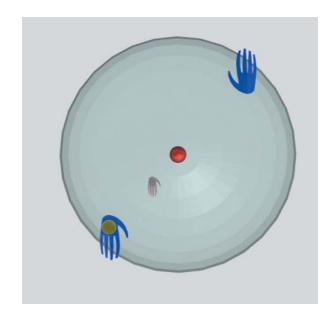

An animation showing how a roto-inversion operates on an object to generate its symmetry-related counterpart.

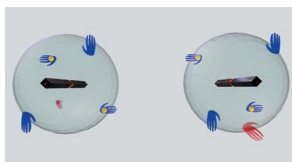

An animation showing how the $2_{1}$ screw axis acts on an object to generate symmetryrelated counterparts.

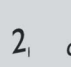

$c$

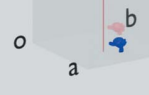

An animation comparing how the $4_{1}$ and $4_{3}$ screw axes act on an object to generate symmetry-related counterparts. The animation shows both operations happening simultaneously side by side to emphasize how the counterclockwise and clockwise arrangements for the objects are generated.

Crystallographic $b$-glide plane: An animation showing how the $b$ glide acts https://skfb.ly/o7NsH on an object to generate symmetry-related objects in subsequent unit cells. interaction. The goal of the problem is for the students to determine the centering and type of each unit cell. A total of 15 problems were created; one for each of the 14 standard Bravais lattice settings with an additional nonstandard monoclinic cell added for additional challenge. These problems are labeled 'Unit Cell Practice $x$ ' where $x$ goes from 1 to 15 , and they can all be found through the unit_cell_ practice tag. Solutions and links to these problems are provided in the SI.

4.3.2. Point-group problems. The point-group problems are designed for students to find symmetry elements and determine the overall point group of a symmetric array of 3D objects. Each problem consists of a set of hands positioned around a sphere with no other symmetry elements present; this problem is meant to integrate well with an exposition of the crystallographic point groups using stereographic projections. One problem exists for each of the 32 point groups, and they are titled 'Stereographic Projection Problem $x$, where $x$ goes from 1 to 32 . They can all be found through the point_ group_practice tag. Solutions and links to these problems are provided in the SI. We have found that a group of 34 students working together can reasonably solve 3-4 of these problems in a $15 \mathrm{~min}$ period.

4.3.3. Space-group problems. The space-group problems each contain a unit cell with a set of symmetry-related objects but no labeled symmetry elements. The goal of these problems is for students to identify the space group represented. All of these problems can easily be found by using the space group_practice tag. These problems have proven to take more time to solve as higher-symmetry unit cells are introduced. For that reason, we recommend starting with triclinic or primitive monoclinic space groups. Currently, problems exist for all triclinic and monoclinic space groups as well as some selected higher-symmetry systems. Solutions and links to all space group problems can be found in the SI.

4.3.4. Block problems. A legacy problem set on point groups for our department consists of a collection of 
Table 1 (continued)

\begin{tabular}{ll}
\hline Description \\
\hline $\begin{array}{l}\text { Symmetry progression for 2/m: } \\
\text { https:/skfb.ly/o7YQJ }\end{array}$ & $\begin{array}{l}\text { An animation showing how the } 2 / m \text { point } \\
\text { group can be constructed by applying } \\
\text { sequential symmetry operations through } \\
\text { three different routes. }\end{array}$
\end{tabular}

Snapshot

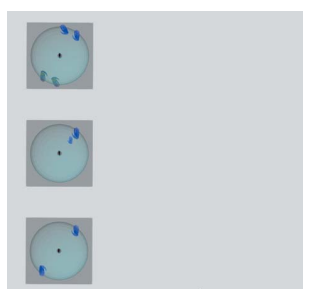

Unit cells

The 14 crystallographic unit A static model showing all 14 unit cells along cells: https://skfb.ly/o7Oxp

with their cell definitions.

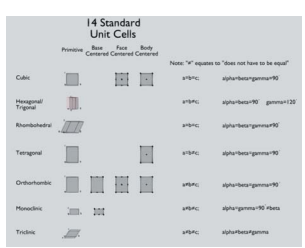

Selected alternative settings for A static model showing the 14 unit cells and unit cells: https://skfb.ly/o7YQO

Bravais lattices (collection): https://skfb.ly/ooY6s

Unit cells for a given lattice: https://skfb.ly/o7YQR three alternative settings for the standard unit cells. Specifically, obverse and reverse are shown for rhombohedral and the body-centered setting for monoclinic.

A collection of models, each one showing one of the 14 standard setting Bravais lattices with cell definitions.
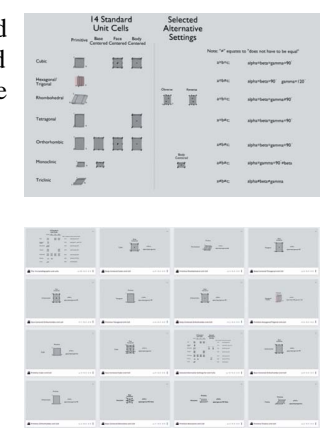

A static model showing how several different unit cells can all be drawn within a single lattice. wooden blocks from the Krantz geological warehouse that were created to depict crystal shapes of various types (https://www.krantz-online.de/en/woodencrystal-models-collection/K15) These wooden blocks were given to students for problem sets or exams, where they were asked to determine the point group of the shape. To preserve and modernize these exercises, as the blocks have become worn through many years of use, and to further distribute them, the shapes of these wooden blocks along with a few other shapes have been recreated in Blender, uploaded to Sketchfab and converted into a 3Dprinting-compatible file format (.stl). Examples of the printed blocks can be seen in Fig. 4. The online versions of these block problems are labeled 'Block $n$ ' where $n$ goes from 1 to 29 , and these names match the $3 \mathrm{D}$ file names found in the SI. All of these problems can easily be found by using the block_problem tag. The solutions and links to these problems are provided in the SI. Each block contains a number cut-out on one face to help differentiate them after they have been printed. This letter should not be considered when trying to determine the point group of the object.

4.3.5. Miller index problems. The Miller index problems contain five models of idealized crystal shapes with multiple facets. Two faces of the shape are labeled as (100) and (010) while the remaining faces are labeled with letters. The goal of this problem is for the students to analyze the shape and determine the crystal system, point group and Miller indices for each of the lettered faces. This assignment is available either via the online model or as a 3D-printed version, which is more satisfying to work with. These problems can be found by using the miller_index tag. The solutions and links to these problems are provided in the SI.

\section{Conclusion}

Blender offers a simple means to generate any $3 \mathrm{D}$ model with a variety of animations. Through collaboration with several experienced crystallographers and students, we have used this tool to create a library of short animations and
Stereographic projection example: https://skfb.ly/o7YRp
An animation showing how the stereographic projection for an orthorhombic shape is generated. These models were inspired by Walter Borchardt-Ott's Crystallography (Borchardt-Ott, 1993).

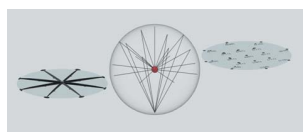


Table 1 (continued)

\begin{tabular}{|c|c|c|}
\hline & Description & Snapshot \\
\hline \multicolumn{3}{|l|}{ Crystallographic point groups } \\
\hline $\begin{array}{l}\text { Crystallographic point groups } \\
\text { (collection): } \\
\text { Tag: Stereographic_- } \\
\text { Projection } \\
\text { (direct links for each model } \\
\text { can be found in the SI) }\end{array}$ & $\begin{array}{l}\text { This collection contains a simple model for } \\
\text { each of the } 32 \text { point groups. All models in } \\
\text { this set include a 3D representation of } \\
\text { their respective symmetry elements. These } \\
\text { models are all titled 'Stereographic } \\
\text { projection: Hermann-Mauguin', where } \\
\text { 'Hermann-Mauguin' is replaced with the } \\
\text { corresponding symbol of the point group. } \\
\text { All models include the tags Hermann- } \\
\text { Maguguin, Stereographic_- } \\
\text { Projection and crystallography_- } \\
\text { education. }\end{array}$ & \\
\hline
\end{tabular}

Crystallographic space groups

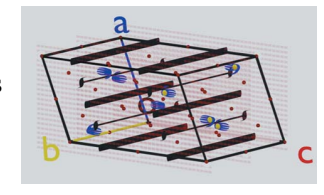

Crystallographic space groups (collection):

Tag: Space_group

(direct links for each model

can be found in the SI)

P2/c symmetry progression: https://skfb.ly/orvnX

This collection contains a simple model for selected space groups. These include all triclinic and monoclinic groups, as well as the more frequently observed highersymmetry groups. All models in this set have a $3 \mathrm{D}$ representation of their respective symmetry elements. A total of 15 space groups were modeled.

An animation showing one possible route to generating the objects in the $P 2 / c$ space group. This animation first applies a twofold rotation, then an inversion, and then the $c$-glide plane.

\section{Miller indices}

Miller indices expansion: https://skfb.ly/orDDR
An animation showing how the faces of a crystal can be expanded to intersect with a coordinate system. These points of intersection are used to determine the face index. static 3D models to aid in the teaching of crystallographic symmetry. We offer downloads to many of these models in the SI of this paper, and we continue to maintain a library of the models via Sketchfab, the free browser-based 3D model viewer. We describe methods by which we incorporate these models into crystallography education through Sketchfab's functionality across handheld smart devices. We will continue to grow this freely accessible library as we garner additional feedback and animation requests from the greater crystallographic community.

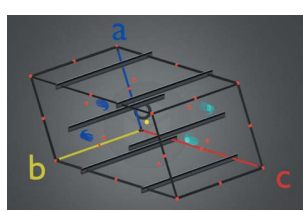

\section{SI}

The included SI contains all models described in this paper. In the main folder is an Excel spreadsheet ('Model Index with links'), which contains a complete list of the models by their point-group symmetry. The folder Stereographic Projection Point Groups Problems contains models for all of the 32 point-group problems, and these models are named by their problem number. The Unit Cell Problems folder contains all unit-cell problems, and these models are named by their problem number. The Unit Cells folder contains all models for individual and composite unit cells, and the name of each model is based on the unit cell it displays.

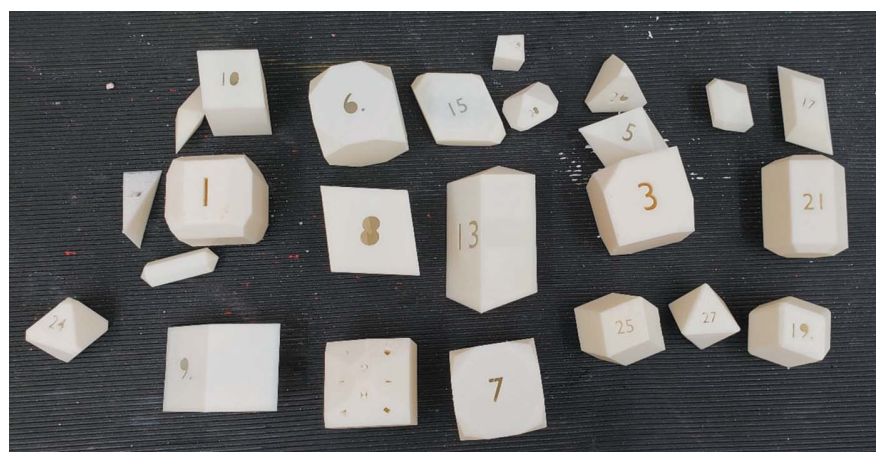

Figure 4

The 3D-printed versions of the blocks. These were printed using ivory color. The 3D-printing files have since been modified to fix inhomogeneity in block size.
An animation of a single-crystal diffractscale with the purpose of demonstrating the size of crystal needed for a data collection. Many parts of the instrument are animated and annotated to provide additional information.

\section{Acknowledgements}

We acknowledge Ilia Guzei, Christine Beavers, Xian Powers, Bruce Noll and the crystallography class of Spring 2021 at UW Madison for their help in creating

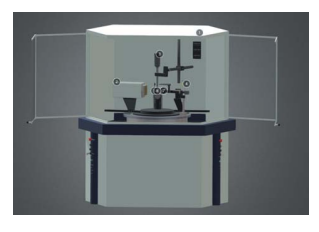

these models. These 3D models (the 'models') are made available as-is for educational purposes, and the Board of Regents of the University of Wisconsin System, its officers, employees and agents ('UW'), makes no representations or warranties of any kind, express, implied, statutory or other, concerning the models. UW expressly disclaims all warranties in connection with use of the models, including but not limited to warranties of fitness for a particular purpose, warranties of merchantability and warranties of non-infringement. Users accept all risk associated with use of the models. By using the models, you acknowledge and agree to the foregoing and waive any and all claims of any kind and description against the Board of Regents of the University of Wisconsin System, 
its officers, employees and agents, arising out of or in connection with your use of the models.

\section{Funding information}

We thank NSF for funding via CHE-1953294, and we thank the NSF-GRFP for funding via DGE-1747503.

\section{References}

Antonoglou, L. D., Charistos, N. D. \& Sigalas, M. P. (2011). Chem. Educ. Res. Pract. 12, 454-468.

Aristov, M. M., Moore, J. W. \& Berry, J. F. (2021). J. Chem. Educ. 98, 3032-3037.

Bazley, I. J., Erie, E. A., Feiereisel, G. M., LeWarne, C. J., Peterson, J. M., Sandquist, K. L., Oshin, K. D. \& Zeller, M. (2018). J. Chem. Educ. 95, 876-881.

Borchardt-Ott, W. (1993). Crystallography. Berlin, New York: Springer.

Brannon, J. P., Ramirez, I., Williams, D., Barding, G. A., Liu, Y., McCulloch, K. M., Chandrasekaran, P. \& Stieber, S. C. E. (2020). J. Chem. Educ. 97, 2273-2279.

Carlisle, D., Tyson, J. \& Nieswandt, M. (2015). Chem. Educ. Res. Pract. 16, 478-517.

Casas, L. (2018). J. Appl. Cryst. 51, 901-908.

Casas, L. (2020). J. Appl. Cryst. 53, 1583-1592.

Casas, L. \& Estop, E. (2015). J. Chem. Educ. 92, 1338-1343.

Chapuis, G. (2011). Crystallogr. Rev. 17, 187-204.

Chen, L., Sun, H. \& Lai, C. (2015). J. Chem. Educ. 92, 1422-1425.

Cushman, C. V. \& Linford, M. R. (2015). J. Chem. Educ. 92, 14151418.

Dagnoni Huelsmann, R., Vailati, A. F., Ribeiro de Laia, L., Salvador Tessaro, P. \& Xavier, F. R. (2018). J. Chem. Educ. 95, 1151-1155.

Duda, M., Rafalska-Łasocha, A. \& Łasocha, W. (2020). J. Chem. Educ. 97, 2169-2174.

Flint, E. B. (2011). J. Chem. Educ. 88, 907-909.

Fuchigami, K., Schrandt, M. \& Miessler, G. L. (2016). J. Chem. Educ. 93, 1081-1084.

Girolami, G. S. (2016). X-ray Crystallography. Mill Valley: Wilsted \& Taylor Publishing Services.

Graef, M. D. (1998). J. Mater. Educ. 20, 31-42.

Grafton, A. K. (2011). J. Chem. Educ. 88, 1281-1282.

Gražulis, S., Sarjeant, A. A., Moeck, P., Stone-Sundberg, J., Snyder, T. J., Kaminsky, W., Oliver, A. G., Stern, C. L., Dawe, L. N., Rychkov, D. A., Losev, E. A., Boldyreva, E. V., Tanski, J. M., Bernstein, J., Rabeh, W. M. \& Kantardjieff, K. A. (2015). J. Appl. Cryst. 48, 19641975.
Grove, N. P., Guerin, N. P., Collins, D. J., López, J. J., Bretz, S. L. \& Zhou, H. C. (2009). J. Chem. Educ. 86, 946.

Hanson, R. M., Prilusky, J., Renjian, Z., Nakane, T. \& Sussman, J. L. (2013). Isr. J. Chem. 53, 207-216.

Hardgrove, G. L. (1997). J. Chem. Educ. 74, 797.

Hitzer, E. M. S., Perwass, C. \& Ichikawa, D. (2010). Geometric Algebra Computing: in Engineering and Computer Science, edited by E. Bayro-Corrochano \& G. Scheuermann, pp. 385-400. Springer London.

International Tables for Crystallography (2016). Vol. A, Space-Group Symmetry, 2nd online ed. Chester: International Union of Crystallography.

Johnston, D. H. (2021). Symmetry Resources at Otterbein University, https://symotter.org/.

Luxford, C. J., Crowder, M. W. \& Bretz, S. L. (2012). J. Chem. Educ. 89, 211-214.

Macrae, C. F., Sovago, I., Cottrell, S. J., Galek, P. T. A., McCabe, P., Pidcock, E., Platings, M., Shields, G. P., Stevens, J. S., Towler, M. \& Wood, P. A. (2020). J. Appl. Cryst. 53, 226-235.

Malbrecht, B. J., Campbell, M. G., Chen, Y. S. \& Zheng, S. L. (2016). J. Chem. Educ. 93, 1671-1675.

Manion, J. G. (2021). CG-Figures, YouTube.

Meier, W. M. (1984). Space Group Patterns. University College Cardiff Press.

Meier, W. M. (2001). 14 Space Group Patterns. University College Cardiff Press.

Penny, M. R., Cao, Z. J., Patel, B., Sil dos Santos, B., Asquith, C. R. M., Szulc, B. R., Rao, Z. X., Muwaffak, Z., Malkinson, J. P. \& Hilton, S. T. (2017). J. Chem. Educ. 94, 1265-1271.

Pettersen, E. F., Goddard, T. D., Huang, C. C., Couch, G. S., Greenblatt, D. M., Meng, E. C. \& Ferrin, T. E. (2004). J. Comput. Chem. 25, 1605-1612.

Rodenbough, P. P., Vanti, W. B. \& Chan, S. W. (2015). J. Chem. Educ. 92, 1960-1962.

Ruiz, G. N. \& Johnstone, T. C. (2020). J. Chem. Educ. 97, 1604-1612. Savchenkov, A. V. (2020). J. Chem. Educ. 97, 1682-1687.

Scalfani, V. F. \& Vaid, T. P. (2014). J. Chem. Educ. 91, 1174-1180.

Southam, D. C. \& Lewis, J. E. (2013). J. Chem. Educ. 90, 1425-1432.

Stróż, K. (1997). J. Appl. Cryst. 30, 178-181.

Stróż, K. (2003). Z. Kristallogr. Cryst. Mater. 218, 642-649.

Schrödinger (2021). pyMOL, https://pymol.org/2/.

Tuvi-Arad, I. \& Blonder, R. (2010). Chem. Educ. Res. Pract. 11, 4858.

Winter, M. (2021). The Orbitron, https://winter.group.shef.ac.uk/ orbitron/. 